\title{
On-line Oil Monitoring and Diagnosis
}

\author{
José Salgueiro 1,* - Gabrijel Peršin² - Jože Vižintin ${ }^{1}$ - Matic Ivanovič ${ }^{3}$ - Boštjan Dolenc ${ }^{4}$ \\ ${ }^{1}$ University of Ljubljana, Faculty of Mechanical Engineering, Slovenia \\ 2 Cranfield University, School of Engineering, Department of Engineering Computing and Cybernetics, UK \\ 3 Institute Jožef Stefan, Department of Systems and Control, Slovenia \\ ${ }^{4}$ University of Ljubljana, Faculty of Electrical Engineering, Slovenia
}

\begin{abstract}
In condition monitoring (CM) of mechanical drives, the analysis of various physical and chemical properties of the operating lubricant can be used to diagnose defects and assess the state of the system. Recent developments in on-line oil condition sensors and advances in signal processing methods have allowed for a system for on-line oil analysis to be developed and applied in the field of predictive maintenance.

The System for On-line Oil Analysis (SOOA) has the ability to measure multiple oil properties of interest and detect faults induced by transients in the acquired signals. Transient detection is based on the cumulative sum of errors (CUSUM) technique, where the error represents the difference between the predicted reference value and the current measured value. Detection of abnormal behaviour, based on transient detection, is followed by fault diagnosis, through integrated assessment of oil properties in real time. The system can operate as a standalone unit with an independent user interface or as a part of a complete integrated diagnostic system, merging oil condition evaluation with vibrational analysis and other techniques.

This paper focuses on the algorithms within SOOA in charge of transient detection and fault diagnosis. The results of SOOA operation are presented through a demonstration of the method in a laboratory environment with two different sets of tests: gear pitting and water contamination.
\end{abstract}

Keywords: predictive maintenance, oil condition monitoring, on-line oil analysis

\section{O INTRODUCTION}

Mechanical systems are composed of different machine elements, with the lubricant being one of the most important machine elements of each rotating machine. The main task of the lubricants is the lubrication of the bearing surfaces in the tribological contacts. It is also responsible for cooling and washing the wear particles away from the contact surfaces. The lubricant in the operating machines is the most important courier of information about the condition of the oil and the machine. During operation, it is difficult to determine the exact time of critical oil degradation and substitution. Because it is difficult to determine when the oil needs changing, periodical (off-line) or permanent (on-line) monitoring of the physical and chemical properties of the lubricant and the presence of wear particles is required [1] and [2]. During the off-line process of oil condition monitoring, samples are taken at pre-defined intervals from random locations in the oil reservoir. Samples are analysed in a laboratory environment using several known techniques, such as total base number (TBN) analysis, viscosity measurements, ferrography, etc. [3] and [4]. The randomness of the sampling location does not, however, ensure that a representative assessment of the machine's actual condition is being performed [5]. In on-line oil condition monitoring, the system for oil analysis is connected directly to the operating reservoir of the mechanical device. In comparison to the off-line methodology, the processes of sampling and analysis are performed without disruption. The generated wear particles on the lubricated elements can also be tracked continuously and with more accuracy, allowing for an early detection of mechanical failures [4] and [5].

As of today, on-line monitoring systems are being designed and tested by the major oil condition online sensors manufactures, but a system with full oil diagnosis coverage is not yet available on the market. An example of a system integrating several individual on-line sensors has been presented in the context of marine diesel engines monitoring [6]. In that study, intelligent software was developed to autonomously analyse the oil properties and diagnose the machine's health state. Concerning oil monitoring in realtime, the challenge lies in the application of a robust automated method for change detection and diagnosis. CUSUM (Cumulative Sum of Errors) is a sequential analysis technique used to detect changes in a given time series assumed to have a statistical distribution of a Gaussian-type centred on 0 and a variance equal to 1 [7]. This approach is the basis for the trend extraction algorithm presented by Charbonnier et al. [8]. Alternatively, Vaswani [9] presents a more complex CUSUM method by applying two different likelihood functions, the expected (negative) log likelihood (ELL) and observation likelihood (OL), which are suitable for slow and fast changes, respectively. Other variations in the CUSUM technique are available 
in Basseville and Nikiforov [7], with theoretical justification.

In Section 1, a multisensory oil monitoring unit (ISU) that is able to measure the fault-indicative oil properties on-line will be presented. This will be followed by a detailed description of intelligent algorithms behind the assessment of lubricant condition. Section 2 is reserved for the experimental evaluation of the SOOA system, including two sets of tests: pitting and water contamination.

\section{DESCRIPTION OF THE SYSTEM FOR ON-LINE OIL ANALYSIS}

\subsection{Architecture}

Fig. 1 shows the system for on-line oil analysis (SOOA), which consists of three main parts: an integrated sensor unit (ISU), an analysis environment (AE), and a maintenance centre (MC).

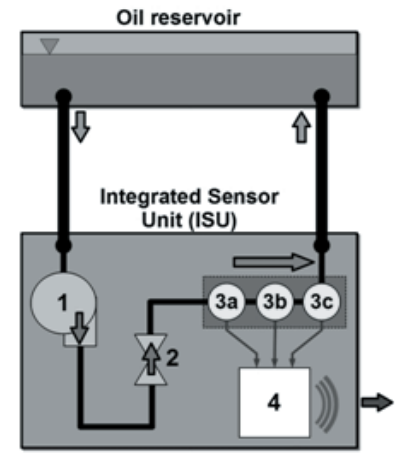

a)
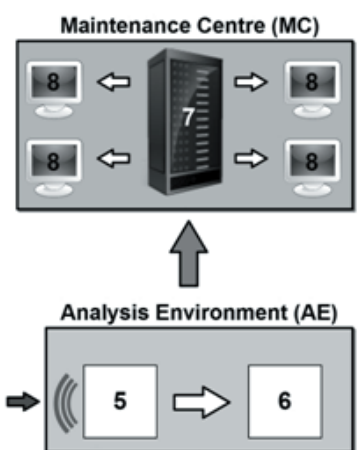

b)

Fig. 1. Architecture of the system for on-line oil analysis:

(1) electromotor pump; (2) hydraulic valve; (3a) to (3c) on-line oil monitoring sensors; (4) smart node; (5) database;

(6) programmable device; (7) server; (8) display screens

\subsection{Integrated Sensor Unit (ISU)}

The Integrated Sensor Unit (ISU) is connected directly to the oil reservoir, Fig. 1a. The ISU includes an electromotor pump (1), a hydraulic security valve (2) and a set of on-line oil monitoring sensors (3a) to $(3 \mathrm{c})$. The sensors are able to measure on-line the oil temperature, relative water content and relative dielectric constant, calculate the generated mass of wear particles, and perform counting of ferrous and non-ferrous particles. The counted particles are divided into five size classes and grouped into two main sets, small and large-sized [10]. For data communication, the ISU unit includes the smart node (SN) unit (4). SN unit is a general programmable platform, responsible for data acquisition and communication with the analysis environment (AE) shown in Fig. 1b. Communication is done wirelessly. Details regarding SN are presented in [11].

\subsection{Analysis Environment}

As shown in Fig. 1b, the AE consists of a database (DB) (5) and programmable device (6), such as a computer or laptop. The data processed in the $\mathrm{AE}$ is also sent to the main server (7) in the MC. The MC's personnel are able to remotely consult the results provided by the SOOA system over digital display screens (8).

The AE is a digital platform designed to evaluate the current oil, wear particles and machine condition based on the measured oil properties and wear particle analysis. In the AE, a graphical user interface (GUI) and a diagnostics module (DM) are included (Fig. 2).

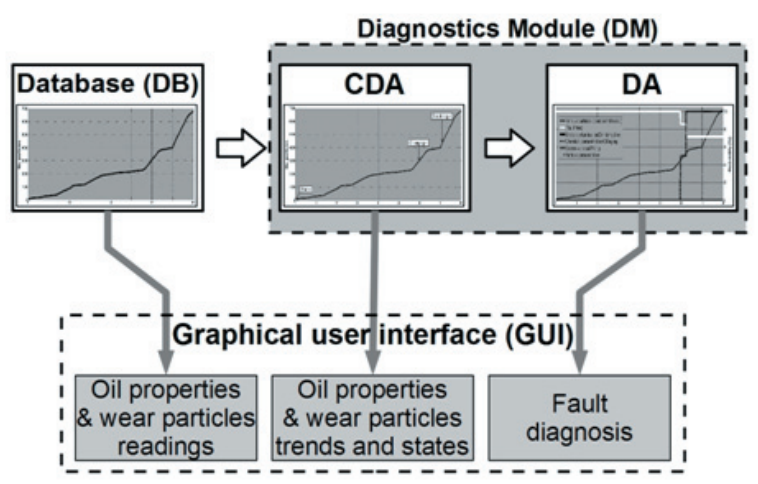

Fig. 2. Architecture of the Analysis Environment

From the DB, the data is channeled for processing at the DM. The main purpose of the DM is to analyse the data from the DM and perform an evaluation of the oil and particles in the oil.

The diagnostics module includes two algorithms: a change detection algorithm (CDA) and a decision algorithm (DA).

\subsubsection{Change Detection Algorithm (CDA)}

The CDA is a tool developed in MATLAB to identify trend changes in a series of data $x\left(t_{i}\right), i=1, \ldots, N$, acquired from on-line sensors and stored in the database. Besides identification of changes, CDA is also able to classify these changes in comparison to the acquired signal's history. 


\subsubsection{Data Acquisition and Normalization}

At any given instant $t=t_{n}$, a vector of the acquired signal, containing the last $N$ samples, is set up, i.e. $X\left(t_{n}\right)=\left[x\left(t_{n-(N-1)}\right), \ldots, x\left(t_{n}\right)\right]$, which is called the current data vector. The current data vector is normalized in the following form $N\left(t_{n}\right)=\left[x^{*}\left(t_{n-(N-1)}\right), \ldots, x^{*}\left(t_{n}\right)\right]$. Each value of the normalized vector $x^{*}\left(t_{j}\right)$ is calculated by using the root mean square $\left(R M S\left(X_{n}\right)\right)$ and average $\left(\overline{X_{n}}\right)$ value as follows:

$$
x^{*}\left(t_{j}\right)=\frac{x\left(t_{j}\right)-\overline{X_{n}}}{\operatorname{RMS}\left(X_{n}\right)} .
$$

\subsubsection{Evaluation of Trends}

Quantitative evaluation of the trend is based on evaluating the linear approximation of the time series $X\left(t_{n}\right)$. A linear trend model is defined as:

$$
f\left(t_{n}\right)=a_{0}+a_{1} t, \quad t \in\left[t_{n-(N-1)}, t_{n}\right] .
$$

In Eq. (2), $a_{0}$ is the value at the origin and $a_{1}$ the slope of the approximation. Both parameters are obtained by the least squares method [12]. The new vector $F\left(t_{n}\right)$ of the approximated values is defined as $F\left(t_{n}\right)=\left[f\left(t_{n-(N-1)}\right), \ldots, f\left(t_{n}\right)\right]$.

Using Eq. (2), the normalized vector $N\left(t_{n}\right)$ is modeled as $F^{*}\left(t_{n}\right)=\left[f^{*}\left(t_{n-(N-1)}\right), \ldots, f^{*}\left(t_{n}\right)\right]$. The linear regression parameters of $F^{*}\left(t_{n}\right)$ are denoted $a_{0}^{*}$ and $a_{1}^{*}$.

\subsubsection{Error and CUSUM Computation}

Having determined $a_{0}^{*}$ and $a_{1}^{*}$, we predict the function values at $t_{n+1}$ using Eq. (2). The extrapolated value $f^{*}\left(t_{n+1}\right)$ from the vector of the approximated values $F(t)$ is compared with the extrapolated value $f_{R}^{*}\left(t_{n+1}\right)$ from the vector of the approximated values $F_{R}(t)$. The vector $F_{R}(t)$ was calculated from the reference vector $X_{R}(t)$. How to obtain the reference vector $X_{R}(t)$ will be detailed later in this section.

An error $e\left(t_{n}\right)$ is obtained as the difference between the two extrapolated values:

$$
e\left(t_{n}\right)=f^{*}\left(t_{n+1}\right)-f_{R}^{*}\left(t_{n+1}\right) .
$$

The cumulative sum (CUSUM) technique, suggested by Charbonnier et al. [8], is used to decide whether the current trend complies with the reference trend. This is done by summing the error values, sample by sample. The CUSUM value $E\left(t_{n}\right)$ is then given by:

$$
E\left(t_{n}\right)=e\left(t_{n}\right)+E\left(t_{n-1}\right) .
$$

The CUSUM value $E\left(t_{n}\right)$ is used to classify how the reference trend and current trend comply with each other. Classification is divided in three stages: acceptable, warning and unacceptable:

- $0 \leq\left|E\left(t_{n}\right)\right| \leq T h_{1}: \quad$ Acceptable;

- $T h_{1}<\left|E\left(t_{n}\right)\right| \leq T h_{2}$ : Warning;

- $\left|E\left(t_{n}\right)\right|>T h_{2}$ : Unacceptable.

When the CUSUM value $E(t)$ exceeds the threshold $T h_{2}$, i.e. the unacceptable stage, a new reference vector $X_{R}(t)$ must be set. The new reference vector $X_{R}(t)$ contains the acquired signal values $x(t)$, when $E(t)$ is between the thresholds $T h_{1}$ and $T h_{2}$, i.e. the warning stage.

\subsubsection{Classification of Trends}

The new reference vector $X_{R}(t)$ which is set with the acquired values $x(t)$, will be modeled according to Eq. (2). The parameter $a_{1}$, Eq. (2), is transformed into reference slope $a_{1 R}$. The current state of the signal is classified with one of the so-called trend states presented in Table 1.

Table 1. Definition of the trend states

\begin{tabular}{lccc}
\hline \multirow{2}{*}{ Trend state } & \multirow{2}{*}{ S } & \multicolumn{2}{c}{ Trend limits } \\
\cline { 2 - 4 } & & from & to \\
\hline "Changing (increasing)" & 3 & $Q^{+}$ & $+\infty$ \\
\hline "Unchanging (increasing)" & 2 & $Q^{-}$ & $Q^{+}$ \\
\hline "Stabilizing (increasing)" & 1 & $T h_{0}$ & $Q^{-}$ \\
\hline "Stable" & 0 & $-T h_{0}$ & $T h_{0}$ \\
\hline "Stabilizing (decreasing)" & -1 & $-Q^{-}$ & $-T h_{0}$ \\
\hline "Unchanging (decreasing)" & -2 & $-Q^{+}$ & $-Q^{-}$ \\
\hline "Changing (decreasing)" & -3 & $-\infty$ & $-Q^{+}$ \\
\hline
\end{tabular}

The new trend state is determined by comparing $a_{1 R}$ and the former reference slope $a_{1 r}$. The parameter $a_{1 r}$ is used to define the trend limits presented in Table 1, using two additional thresholds $T h_{Q}$ and $T h_{0}$ ("Stable" and "Unchanging" thresholds, respectively). $T h_{Q}$ can be set in terms of percentages. $T h_{0}$ is an absolute parameter which is set in CDA. $T h_{0}$ should be measured and adjusted according to the oil property or wear particle number. $Q^{-}$and $Q^{+}$are the limits of the "Unchanging" trend state and are defined as follows:

$$
\begin{aligned}
& Q^{+}=a_{1 r}+0.5\left(a_{1 r} \cdot T h_{Q}\right), \\
& Q_{-}=a_{1 r}-0.5\left(a_{1 r} \cdot T h_{Q}\right) .
\end{aligned}
$$

The possible transitions from one trend state to another have their restrictions. Fig. 3 shows which 
transitions are possible, depending on the original trend state.

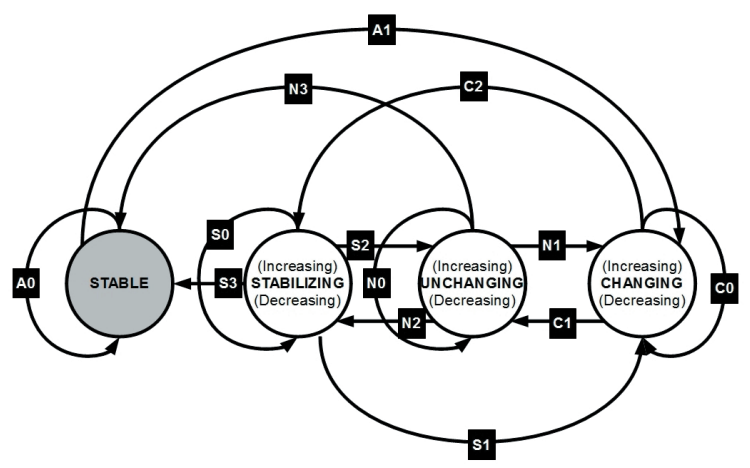

Fig. 3. Trend state transition diagram

The different transitions are coded by a letter and an index number (Fig. 3). The letter depends on the original trend state: A - "Stable"; S - "Stabilizing"; $\mathrm{N}$ - "Unchanging"; C - "Changing". The index 0 indicates that no change in the trend state occurred. When the indexes are larger than 0 this indicates that the new trend state differs from the previous one.

\subsubsection{State-Transition Vector Definition}

The state-transition (ST) vector is defined as $\mathbf{S T}=[s t(1), s t(2), \ldots, s t(M)]$. The $s t$ values, i.e. statetransition indicators, are the trend state values $s$ presented in Table 1 that are relevant for signal shape recognition. For recognition to be possible via this method, repetition of $s t$ values within the ST vector are to be avoided - the trend state value $s\left(t=t_{n}\right)$ will only be added to the $\mathbf{S T}$ vector if it differs from the previous value $s t(\mathrm{M})$, i.e. if the transition index differs from 0 , see the diagram in Fig. 3:

if $s\left(t=t_{n}\right) \neq s t(M)$ then $\mathbf{S T}=\left[s t_{1}, s t_{2}, \ldots, s t_{M}, s\left(t_{n}\right)\right] .(6)$
In order to eliminate false alarms due to temporary variations in the targeted oil property or wear particle count, the output of the $s\left(t_{n}\right)$ value as the latest statetransition value $s t$ is purposely delayed. The $s t$ value is only kept if $s\left(t_{n}\right)$ remains unchanged for a certain pre-chosen amount of time.

If the signal returns to a "Stable" state, then the ST vector is reset:

$$
\text { if } s\left(t=t_{n}\right)=0 \text { then } \mathbf{S T}=[0] .
$$

\subsubsection{Decision Algorithm (DA)}

The DA is a tool developed in MATLAB which uses the information provided by CDA to recognize modeled transient shapes of the signals, which are fault indicative and perform fault diagnosis. DA includes a fault-modes table (FMT), shape recognition and fault diagnosis modules (Fig. 4).

The decision algorithm goes through all fault cases in the FMT to determine the probability of each. For the specific fault, DA checks the influence of all oil properties individually.

\subsubsection{FMT Table}

The FMT is a knowledge database that connects faulty events to identifiable transients for each individual oil property.

Table 2 shows which oil properties are indicative of a specific fault and how they evolve over time. Note that a fault-free case is included and can be used to assess if the mechanical system being diagnosed is performing under nominal conditions, i.e. if all oil properties are stable. A fault modes table has been established based on the theory and experimental work presented in [1], [5], [10] and [14] and predicts
SHAPE RECOGNITION

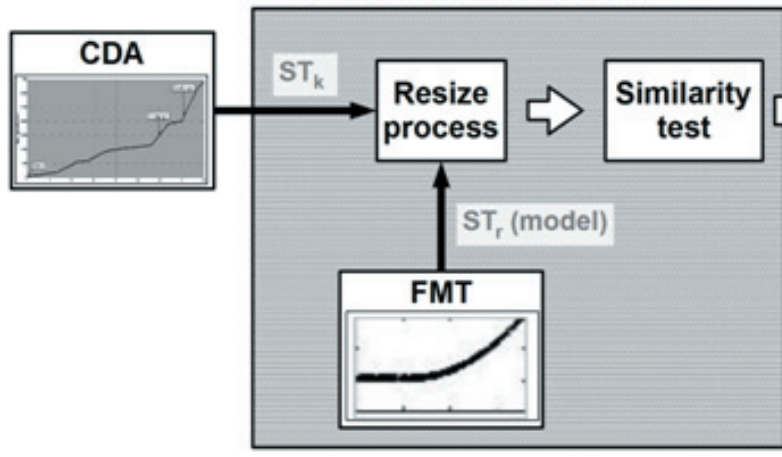

FAULT DIAGNOSIS (for fault $j$ )

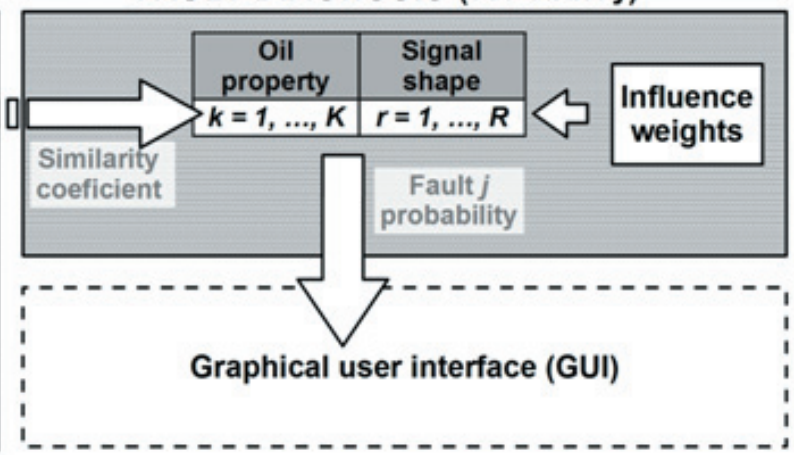

Fig. 4. Decision algorithm's process of fault diagnosis 
Table 2. Fault-modes table

\begin{tabular}{|c|c|c|c|c|c|c|c|}
\hline \multirow[b]{2}{*}{ Fault } & \multicolumn{7}{|c|}{ Oil property } \\
\hline & Temperature & $\begin{array}{c}\text { Rel. water } \\
\text { content }\end{array}$ & $\begin{array}{l}\text { Rel. dielectric } \\
\text { constant }\end{array}$ & $\begin{array}{c}\text { Ferrous part. } \\
\text { count } \\
\text { (small-sized) }\end{array}$ & $\begin{array}{c}\text { Ferrous part. } \\
\text { count } \\
\text { (large-sized) } \\
\end{array}$ & $\begin{array}{c}\text { Non-ferrous } \\
\text { part. count } \\
\text { (small-sized) }\end{array}$ & $\begin{array}{c}\text { Non-ferrous } \\
\text { part. count } \\
\text { (large-sized) } \\
\end{array}$ \\
\hline (Eault_froo) & & & & & & & \\
\hline & & & & & & & \\
\hline $\begin{array}{l}\text { Water } \\
\text { contamination/ } \\
\text { Condensation }\end{array}$ & (No influence) & & (No influence) & (No influence) & (No influence) & (No influence) & (No influence) \\
\hline $\begin{array}{l}\text { Chemical } \\
\text { contamination/ } \\
\text { Oil aging }\end{array}$ & (No influence) & (No influence) & & (No influence) & (No influence) & (No influence) & (No influence) \\
\hline $\begin{array}{l}\text { Excessive wear/ } \\
\text { Pitting }\end{array}$ & (No influence) & (No influence) & (No influence) & & & (No influence) & (No influence) \\
\hline $\begin{array}{l}\text { Particle } \\
\text { contamination }\end{array}$ & (No influence) & (No influence) & (No influence) & (No influence) & (No influence) & & \\
\hline
\end{tabular}

four possible fault events and one additional fault-free case.

\subsubsection{Shape Recognition}

From the CDA signal processing of oil property $k$, the corresponding state-transition vector $\mathbf{S T}_{k}$ is determined. From the FMT, a model for the signal shape $r$ is also provided in the form of a statetransition vector $\mathbf{S T}_{r}$. In order for the similarity test to be possible, these vectors have to be resized to the same length.

If $\mathbf{S T}_{k}$ and $\mathbf{S T}_{r}$ are composed of $N$ and $M$ elements, respectively, then these are resized into two new $L$-elements $(L=N \times M)$ vectors. The scaling process is done by replicating and proportionally distributing the elements in the original ST into a new resized $\mathbf{S T}^{\prime}$ vector.

A sequence similarity test is then performed. For that, a binary scoring function is defined such that:

$$
\sigma\left(s t_{k}(l), s t_{r}(l)\right)=\left\{\begin{array}{ll}
0, & s t_{k}(l) \neq s t_{r}(l) \\
1, & s t_{k}(l) \neq s t_{r}(l)
\end{array} .\right.
$$

According to [15], the value of the alignment between the vectors $\mathbf{S} \mathbf{T}^{\prime}{ }_{k}$ and $\mathbf{S} \mathbf{T}_{r}^{\prime}$ can be calculated by summing all scoring results from Eq. (8):

$$
A=\sum_{l=1}^{L} \sigma\left(s t_{k}(l), s t_{r}(l)\right) \text {. }
$$

In the end, the similarity coefficient is the ratio between the vector alignment value $A$ and the number of elements of any of the resized vectors:

$$
\operatorname{sim}\left(S T_{k}, S T_{r}\right)=\frac{A}{L}
$$

\subsubsection{Fault Diagnosis}

The result for the similarity test is inputted into the fault diagnosis process (Fig. 4). For each fault event defined in the FMT we perform a number $K$ of similarity tests. $K$ is the number of acquired signals from the on-line sensors (Fig. 4). The fault $j$ probability is calculated with the following equation:

$$
p(j)=\sum_{k=1}^{K} \operatorname{sim}_{k}\left(S T_{k}, S T_{r}\right) \times w_{k},
$$

Where $w_{k}$ is the influence weight of oil property $k$ for the specific fault $j$. If all oil properties are equally influential in the identification of fault $j$, then $w_{k}=1 / k$. If not, then the summation of all $w_{k}$ must still be equal to 1 :

$$
\sum_{k=1}^{K} w_{k}=1
$$

The results for all fault probabilities $p(j)$ are then shown on the Graphical User Interface (GUI) as a percentage (Fig. $5 b$ ). 

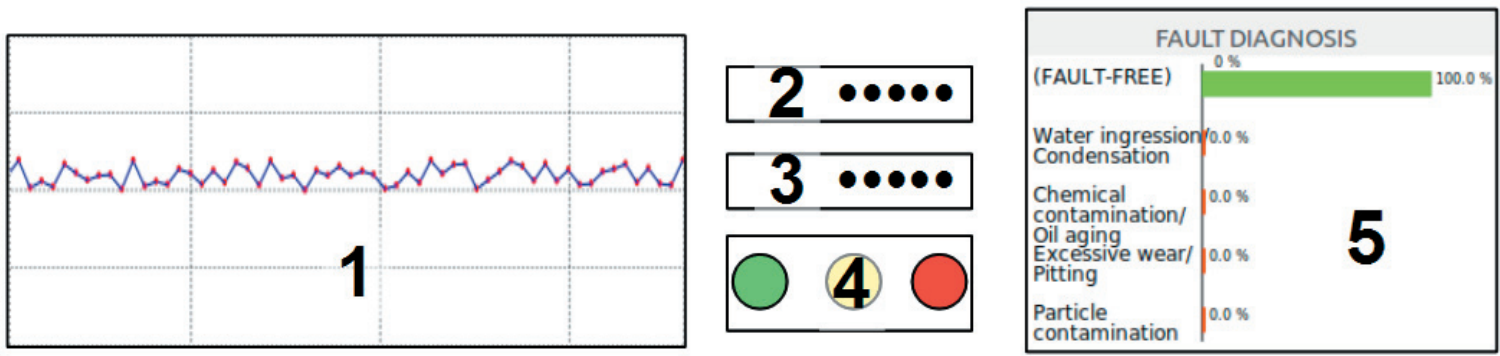

Fig. 5. Graphical user interface; (1) acquired signal plot, (2) last value of the acquired signal,

(3) rate of change for the acquired signal, (4) light indicator, (5) fault diagnosis subsection

\subsubsection{Graphical User Interface (GUI)}

The graphical user interface is a visual environment for the AE. The GUI is divided into two parts or subsections (Fig. 5).

Element (1) in Fig. 5 shows the plot of the acquired signals for oil properties and wear particles measured by the on-line sensors. The last value (2) and rate of change (3) of the acquired signal are also presented in this subsection. Concerning the traffic light indicator (4), the final status ("Good" green, "Warning" - yellow and "Critical" - red) is determined for each oil property and wear particle count.

Fig. 5 also presents element (5), which is the fault diagnosis subsection showing a monitored machine under nominal conditions of operation. If any fault in the tribological contact is evolving, the "fault-free" percentage will drop in favor of an increase in any probability of fault occurring.

\subsection{Maintenance Center}

The MC is composed of a group of maintenance experts that take decisions based on the SOOA's evaluation of condition of the oil and identification of faults. In the MC, the experts can control the oil condition monitoring process by consulting the visual elements presented in the GUI (Fig. 5). The SOOA system also provides reports about the on-line oil monitoring process upon occurrence of a faultindicative event.

The data and diagnostic results are stored in the DB and are available to be included in the local computerized maintenance management system (CMMS). With the CMMS, the maintenance managers can compare the machinery documentation with the on-line oil condition monitoring information. With this, the experts at the MC can make a final decision about the state of health of the machine and schedule the necessary interventions.

\section{EVALUATION OF THE ON-LINE OIL ANALYSIS SYSTEM}

In order to test the efficiency of the system, experiments were performed on a laboratory test rig to observe the following faults: pitting (Section 2.2) and water contamination (Section 2.3).

\subsection{Experimental Setup}

The laboratory experimental setup consists of a synchronous electric motor (1) and a brake-generator (3) that imposes resistive torque. A single-stage gearbox (2), with a transmission ratio of 1.5 , connects the input and output shafts. Shafts are coupled to the motor and generator by two elastic and one fixed coupling. The ISU unit (4) hydraulic input and output were connected directly to the gearbox (Fig. 6).

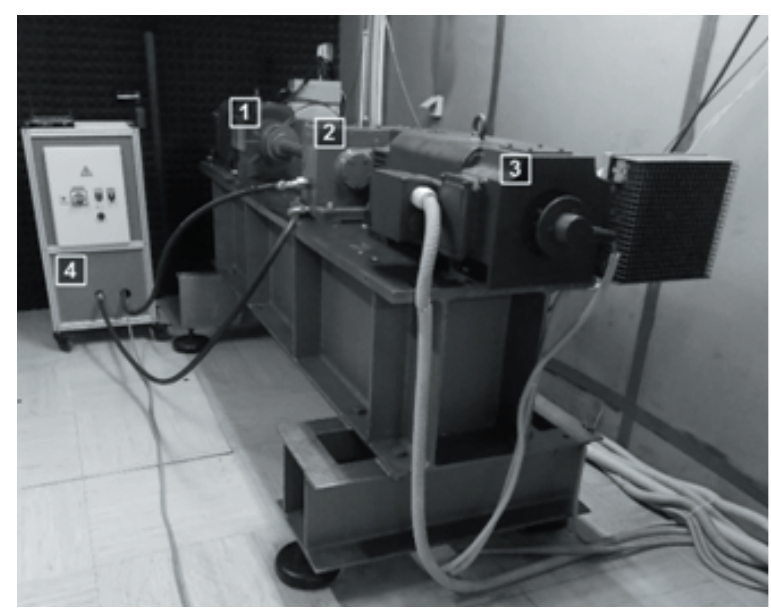

Fig. 6. Experimental setup

The synchronous electric motor has a rated power of $12.7 \mathrm{~kW}$ and speed of $1470 \mathrm{rpm}$. The brakegenerator has a $20.2 \mathrm{~kW}$ rated power and $110 \mathrm{Nm}$ 
maximum torque. Inside the gearbox, a pair of nitrated spur gears (DIN 42CrMo4) with 16 and 24 teeth was installed. Two liters of gear oil were poured inside the gearbox for each test.

Before starting the monitoring process, the CDA's input parameters were inserted into the DM as presented in Table 3.

Table 3. Input parameters for the Change Detection Algorithm (CDA)

\begin{tabular}{|c|c|c|c|c|c|}
\hline \multirow{2}{*}{ Oil property } & \multirow{2}{*}{$\begin{array}{c}\text { Data } \\
\text { window } \\
\text { length } \\
\text { [h] }\end{array}$} & \multicolumn{2}{|c|}{$\begin{array}{l}\text { CUSUM } \\
\text { thresholds }\end{array}$} & \multicolumn{2}{|c|}{$\begin{array}{l}\text { Trend state } \\
\text { thresholds }\end{array}$} \\
\hline & & $T h_{1}$ & $T h_{2}$ & $T h_{0}$ & $\begin{array}{l}T h_{Q} \\
{[\%]}\end{array}$ \\
\hline $\begin{array}{l}\text { Relative water } \\
\text { content }\end{array}$ & 3 & $5 \%$ & $50 \%$ & $0.5 \% / h$ & $25 \%$ \\
\hline $\begin{array}{l}\text { Ferrous particle } \\
\text { count (small-sized) }\end{array}$ & 6 & $\begin{array}{c}20 \\
\text { particl. }\end{array}$ & $\begin{array}{c}200 \\
\text { particl. }\end{array}$ & $\begin{array}{c}100 \\
\text { particles/h }\end{array}$ & $25 \%$ \\
\hline $\begin{array}{l}\text { Ferrous particle } \\
\text { count (large-sized) }\end{array}$ & 6 & $\begin{array}{c}20 \\
\text { particl. }\end{array}$ & $\begin{array}{c}200 \\
\text { particl. }\end{array}$ & $\begin{array}{c}20 \\
\text { particles/h }\end{array}$ & $25 \%$ \\
\hline
\end{tabular}

All parameters presented in Table 3 were determined from observation and testing of the data from previous experiments on the experimental setup. These are the parameters we recommend for on-line monitoring of gear oil for this specific experimental setup. These parameters can be changed from the GUI at any time by the maintenance expert once the type of lubricant or the machine elements have been changed by the maintenance intervention.

\subsection{Pitting Test}

The pitting test was conducted for 120 hours. The sampling time was 1 minute for every analysed signal. The test was conducted under time varying torque conditions to test the influence of load variation in promoting pitting phenomena. The torque was set to vary in steps of $33 \%$ of the motor's maximum torque every 7 hours, as shown in the profile presented in Fig. 7. The motor speed was set to a constant $1296 \mathrm{rpm}$.

The acquired data was analysed and presented in the GUI (Fig. 5). To present the results of the pitting test from GUI in a more suitable form, we prepared the plots as shown in Fig. 8. The fine black lines in Fig. $8 \mathrm{a}$ and $\mathrm{b}$ show the number of small and largesized ferrous particles generated during the pitting test.

During the running-in period, from 0 to 56 hours, the small and large-sized particles increased very slowly. Following the running-in period, both sizeclass particles started to increase faster (Fig. 8a and b).
In the running-in period, the fault-free probability is $100 \%$, meaning that no faults occurred.

After this period, CDA indicated an increase in the number of small and large-sized wear particles (Fig. 8, "Changing +"). CDA's indication transmitted to DA which calculated the fault-free probability and excessive wear in the tribological contact. The probability of fault-free then decreased from 100 to $72 \%$ (white bold line). On the other hand, the probability of excessive wear increased from 0 to $50 \%$ first and to $100 \%$ after (black dashed line). The pitting fault was fully indicated by the diagnostics module after 62 hours of the test run. Pitting on the gear teeth surface was confirmed with visual inspection at the $72^{\text {nd }}$ hour.

The following variations in CDA's output and DA's calculation of fault-free and excessive wear probability are resulted from the variations in imposed torque, Fig. 7.

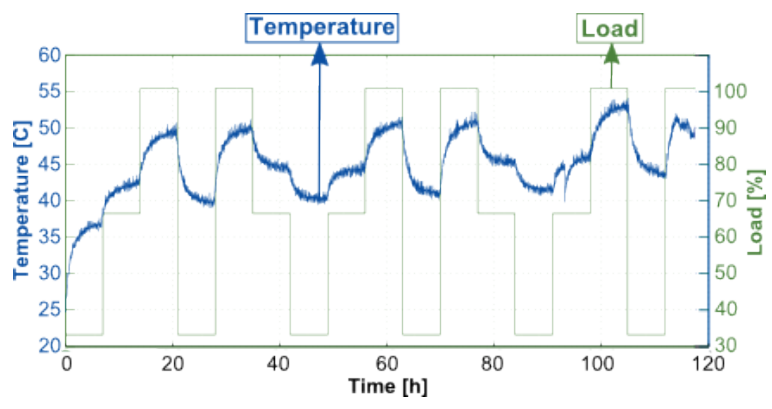

Fig. 7. The time varying torque profile and temperature evolution

\subsection{Water Contamination Test}

This test was performed in order to observe and indicate the influence of water contamination in the operating gear oil. The water contamination test lasted for 60 hours. Motor speed was set to a constant 1000 $\mathrm{rpm}$ and the torque to constant $28 \mathrm{Nm}$. After 5 hours of the test run, $1 \mathrm{ml}(500 \mathrm{ppm})$ of tap water was dropped through an inlet socket into the gearbox (white circle in Fig. 9).

The water ingression immediately produced an abrupt change in the relative water content. After this change, the relative water content slowly decreased until 60 hours into test run (Fig. 9).

After approximately 6 hours, the fault-free indication dropped from 100 to $86 \%$ (white bold line). At the same time, DA indicates an increase in the water contamination probability, from 0 to $50 \%$ and then to $100 \%$ (black bold line). This fully confirms that the gear oil was contaminated with water. 


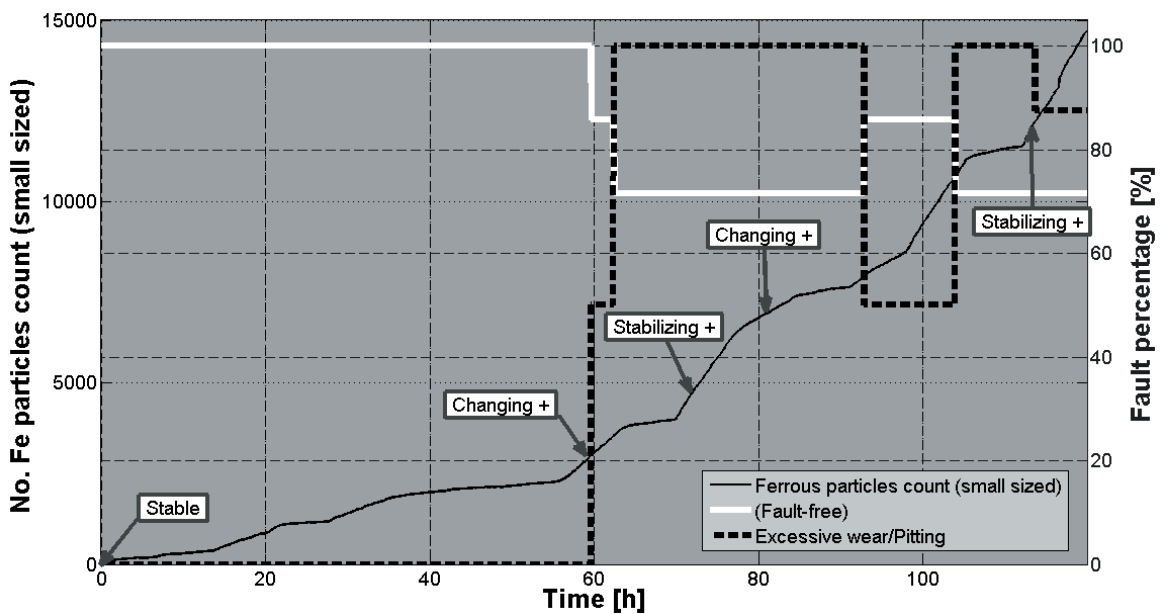

a)

Time [h]

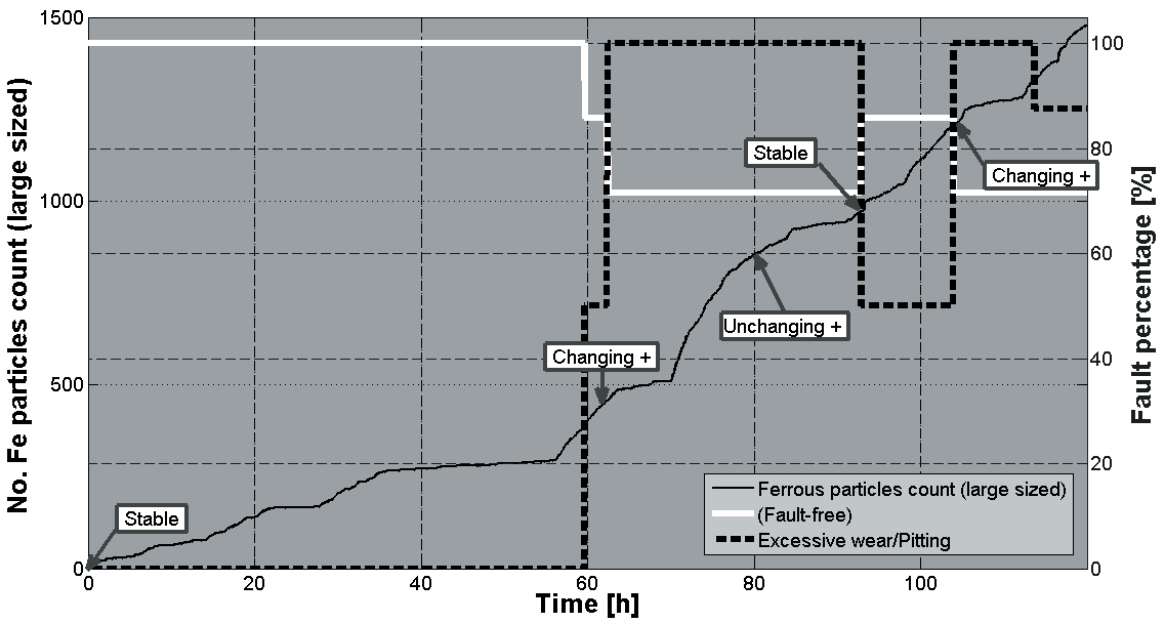

Fig. 8. Results of the pitting experiment; a) Ferrous particle count (small-sized), b) Ferrous particle count (large-sized)

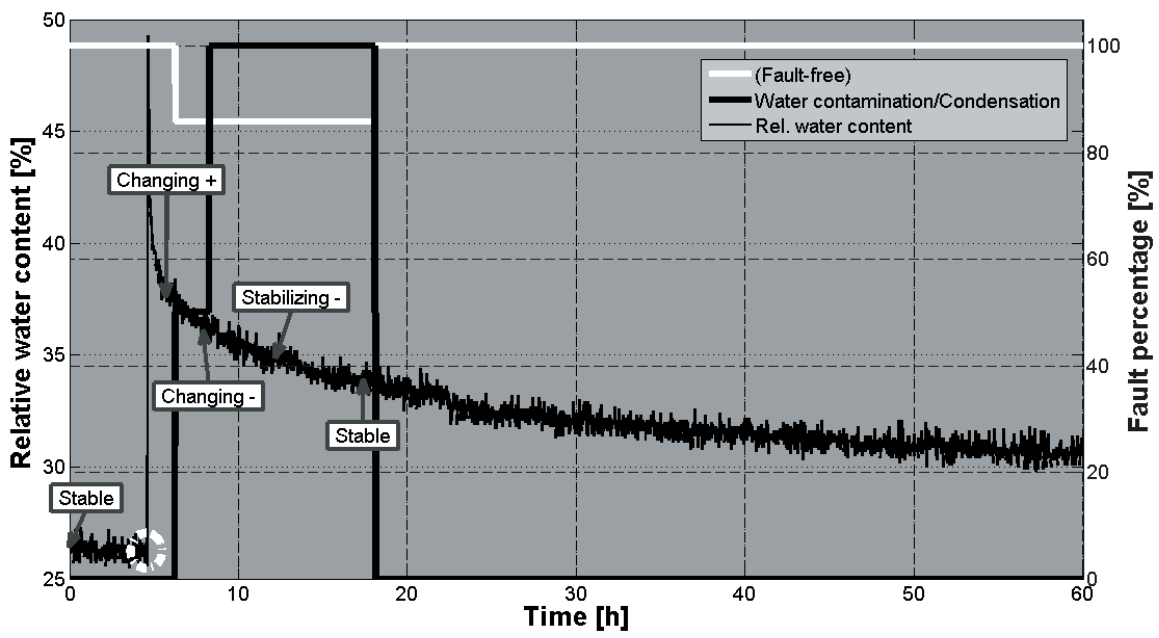

Fig. 9. Results from the water contamination experiment

After 18 hours of operation, CDA detects that the relative water content readings are stable. As a consequence of this, the DA fault-free results increase back to $100 \%$ and the water contamination probability falls to $0 \%$. 


\section{CONCLUSIONS}

The SOOA system can monitor physical and chemical gear oil properties and generation of wear particles, in terms of number, size and mass. Based on the monitoring of oil properties and generated particles, the SOOA system makes a fault diagnosis through integration of individual assessments.

An analysis environment for communication with the operator/maintenance centre was developed and tested under live conditions. The evaluation of the diagnostic module was achieved through experimental tests in a laboratory environment. The response of the diagnostic algorithms was achieved in appropriate time, proving that the methods used for transient identification are able to successfully pinpoint the occurrence of the modeled failure events.

The SOOA can operate as a separate system or as a part of a larger integrated diagnostic system. In this integrated diagnostic system, full machinery diagnosis can be achieved by merging oil condition monitoring with vibrational and acoustic signal analysis and by monitoring the environment and contact temperature.

\section{ACKNOWLEDGEMENTS}

This work was carried out within the framework of an Applied Project supported by the Slovenian Agency for Research and Development (ARRS) and the Competence Centre for Advanced Control Technologies. It was partly financed by the Republic of Slovenia, Ministry of Higher Education, Science and Technology and the European Union (EU) European Regional Development Fund within the Operational Programme for Strengthening Regional Development Potentials for the Period 2007 to 2013.

\section{REFERENCES}

[1] Vižintin, J., Kambič, M., Lipušček, I., Hudnik, V. (1995). Application of Wear Particle Analysis to Condition Monitoring of Rotating Machinery in Iron and Steel Works. Lubrication Engineering, vol. 51, no.5, p. 389-393.

[2] Gonçalves, A., Cunha R., Lago, D. (2007). Vibration and wear particles analysis in a test stand. Industrial Lubrication and Tribology, vol. 59, no. 5, p. 209-216, DOI:10.1108/00368790710776793.

[3] Gonçalves, A., Almeida, L., Mathias, M. (2010). Wear Particle Classifier System Based on an Artificial Neural Network. Strojniški Vestnik - Journal of Mechanical Engineering, vol. 56, no. 4, p. 284-288.

[4] Vähäoja, P.O., Pikkarainen, H.V.S. (2010). Trends in industrial oil analysis - a review. International
Journal of Condition Monitoring, vol. 1, no. 1, p. 4-8, DOI:10.1784/204764211798089057.

[5] Kržan, B., Vižintin, J. (2008). On-line wear and lubricant condition monitoring. Proceedings of the 5th International Conference on Condition Monitoring and Machinery Failure Prevention Technologies, Edinburgh, (CD-ROM).

[6] Knowles M., Baglee D. (2012). Condition Management of Marine Lube Oil and the Role of Intelligent Sensor Systems in Diagnostics. Proceedings of the 25th International Congress on Condition Monitoring and Diagnostic Engineering (COMADEM 2012), vol. 1, p. 59-68, Huddersfield.

[7] Basseville, M., Nikiforov, I.V. (1993). Detection of Abrupt Changes: Theory and Application. PrenticeHall, Englewood Cliffs, New Jersey.

[8] Charbonnier, S., Garcia-Beltan, C., Cadet, C., Gentil, S. (2005). Trends extraction and analysis for complex system monitoring and decision support. Engineering Applications of Artificial Intelligence, vol. 18, no. 1, p. 21-36, DOI:10.1016/j.engappai.2004.08.023.

[9] Vaswani, N. (2005). The modified CUSUM algorithm for slow and drastic change detection in general HMMs with unknown change parameters. Proceedings of the IEEE International Conference on Acoustics, Speech and Signal Processing (ICASSP '05), vol. 4, p. iv/701iv/704.

[10] Peršin, G., Salgueiro, J., Vižintin, J., Juričić, Đ. (2012). A system for automated online oil analysis. Insight - Non-Destructive Testing and Condition Monitoring, vol. 54, no. 8, p. 428-432, DOI:10.1784/ insi.2012.54.8.428.

[11] Ivanovič, M., Boškoski, P., Juričić, Đ. (2012). An environment for efficient design and implementation of condition monitoring systems for mechanical drives. Proceedings of the 9th International Conference on Condition Monitoring and Machinery Failure Prevention Technologies, London (CD-ROM).

[12] Wasserman, L. (2004). All of Statistics: A Concise Course in Statistical Inference. Springer Science+Business Media, Inc., New York.

[13] Holmerg, K., Helle, A., Halme, J. (2008). Machinery reliability future issues: Performance prognostics and e-Maintenance. Proceedings of the Conference on Technical Diagnostics, Lubricants and Fuels, Ljubljana, p. 3-15.

[14] Byington, C.S., Schalcosky, D.C. (2000). Advances in Real Time Oil Analysis. Machinery Lubrication, from http://www.machinerylubrication.com/Read/138/realtime-oil-analysis accessed on 2012-09-03

[15] Tompa, M. (1996). Courses in Computer Science and Engineering, CSE 590BI: Algorithms in Molecular Biology. Lecture 2 - Sequence Similarity. University of Washington, Computer Science and Engineering, from http://www.cs.washington.edu/education/courses/ cse527/96wi/, accessed on 2012-09-14 